SCIPP 93/33

February 1, 2008

\title{
Detecting Technibaryon Dark Matter
}

\author{
John Bagnasco, Michael Dine, and Scott Thomas \\ Santa Cruz Institute for Particle Physics \\ University of California, Santa Cruz, CA 95064
}

\begin{abstract}
The technibaryon constitutes a possible dark matter candidate. Such a particle with electroweak quantum numbers is already nearly ruled out as the dominant component of the galactic dark matter by nuclear recoil experiments. Here, the scattering of singlet technibaryons, without electroweak quantum numbers, is considered. For scalar technibaryons the most important interaction is the charge radius. The scattering rates are typically of order $10^{-4}(\mathrm{~kg} \mathrm{keV} \mathrm{day})^{-1}$ for a technicolor scale of $1 \mathrm{TeV}$. For fermionic technibaryons the most important interaction is the magnetic dipole moment. The scattering rates in this case are considerably

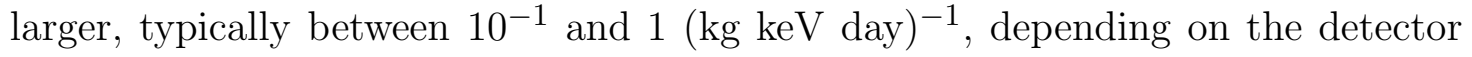
material. Rates this large may be detectable in the next generation of nuclear recoil experiments. Such experiments will also be sensitive to quite small technibaryon electric dipole moments.
\end{abstract}

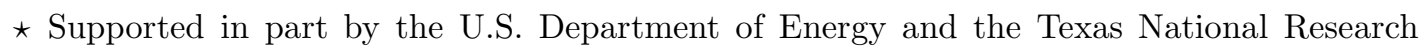
Laboratory Commission under grant numbers RGFY 93-263 and RGFY 93-330. 


\section{Introduction}

Technicolor and supersymmetry are the most attractive alternatives for electroweak symmetry breaking. Each also provides an interesting class of dark matter candidates. The idea that the lightest supersymmetric particle might be the dark matter has been widely explored. For a substantial range of the SUSY parameter space, $\Omega \sim 1$, where $\Omega$ is the average density divided by the critical density. ${ }^{[1]}$ Technicolor is currently the less popular alternative; there are many obstacles to developing a workable model, much less an attractive one. However, it can naturally give rise to a suitable dark matter candidate, the technibaryon. ${ }^{[2]}$ In most technicolor models, there is a technibaryon symmetry analogous to ordinary baryon number. At low temperatures the lightest technibaryon is very nearly stable. If technibaryon number possesses an $S U(2)_{L}$ anomaly, some linear combination of baryon and technibaryon numbers will be anomaly free. An initial net baryon number in the very early universe is then converted to roughly equal baryon and technibaryon asymmetries by electroweak sphaleron processes. ${ }^{[3]}$ Also, baryon production at the electroweak phase transition will lead to roughly equal technibaryon production ${ }^{[4]}$ These mechanisms naturally give $\Omega_{B} / \Omega_{b} \sim \mathcal{O}\left(\Lambda_{T C} / m_{n}\right)$ times a model-dependent suppression, where $\Lambda_{T C}$ is the technicolor scale, $m_{n}$ the nucleon mass, and $\Omega_{B}$ and $\Omega_{b}$ are for the technibaryons and baryons respectively.

If technibaryons do constitute the dark matter, the question of direct detection arises. Searches for anomalously heavy isotopes ${ }^{[5]}$ and astrophysical constraints ${ }^{[6]}$ exclude electrically charged and strongly interacting particles. Experiments sensitive to nuclear recoil by elastic scattering rule out particles with weak isospin up to a mass of a few $\mathrm{TeV}$ as the dominant component of the galactic dark matter. ${ }^{[7]}$ Singlet technibaryons, without electroweak quantum numbers, will be considerably harder to detect in recoil experiments. ${ }^{[8]}$ Most discussions of the detection of such particles have focussed on residual color interactions. Nussinov suggested that these might be strong enough to allow detection. ${ }^{[9]}$ Subsequently, Chivukula et. al. ${ }^{[10]}$ by examining possible effective operators which would describe such interactions, argued that this is not the case. However, these treatments ignored electromagnetic interactions, associated with possible charge radii and magnetic moments.

In this letter a systematic analysis of the effective operators leading to elastic scattering of singlet technibaryons on nuclei is presented. Since the technibaryon carries a conserved quantum number for a continuous symmetry (technibaryon number), the fields are either complex bosons or Dirac fermions. This allows additional effective operators not possible for real bosons or Majorana fermions. Depending on the details of the technicolor theory, the lightest technibaryon may 
be either bosonic or fermionic. ${ }^{\dagger}$ Each case will be considered separately. For scalar bosonic technibaryons the most important operator turns out to be the charge radius. This couples coherently to the nuclear charge. The scattering rates are quite small, however, being suppressed by four powers of the technicolor scale. The scattering rates are considerably larger for fermionic technibaryons. Here the leading operator is the magnetic moment. Just as for QCD, technifermions are expected to have (relatively) large magnetic moments, of the order of the Dirac moment for a comparable charged particle. ${ }^{[9]}$ This moment couples to the nuclear magnetic moment and coherently to the nuclear charge. The scattering rate is suppressed by only two powers of the technicolor scale. If technifermions comprise the galactic dark matter, the scattering rates are large enough to be detected in the next generation of experiments. The fermionic case possesses another interesting feature. It is possible for fermionic technibaryons to have an electric dipole moment. Such a CP violating effect would be expected to be suppressed by a large scale associated with $\mathrm{CP}$ violation, $\Lambda_{C P}$, e.g. some extended technicolor scale. Even so, this moment couples coherently to the nucleus with a large infrared enhancement. If $\Lambda_{C P}$ is much larger than $\Lambda_{T C}$ however, the magnetic moment still gives the dominant scattering.

Although the analysis presented here is for technibaryons, it is generally applicable to any dark matter particle which carries a quantum number of a continuous symmetry, but is a singlet under the standard model gauge groups. ${ }^{[4]}$ The effective operators and resulting scattering rates for scalar and fermionic technibaryons are discussed in the next two sections. The final section addresses the question of detectability and the possibility of experimentally distinguishing technibaryons from the supersymmetric neutralino.

$\dagger$ Singlet fermionic technibaryons require singlet or vector representations of $S U(2)_{L}$. We thank R. S. Chivukula for this comment. 


\section{Scalar Technibaryons}

The coupling of a singlet technibaryon to nuclei can be regarded as arising from effective operators defined just above the hadronic scale, $\mathcal{O}(1 \mathrm{GeV})$. At this scale the relevant degrees of freedom are the photon, gluon, and light quarks. The effective operators are bilinears in the technibaryon field multiplied by the light field operators. The light field operators are required to be $S U(3)_{C} \times U(1)_{Q}$ invariant and Lorentz covariant. First consider the coupling to the photon and gluons. At dimension three the only operator involving these gauge bosons which can couple to a singlet scalar technibaryon is

$$
\partial_{\mu} F^{\mu \nu}
$$

where $F^{\mu \nu}$ is the electromagnetic field strength. Using the equation of motion $\partial_{\mu} F^{\mu \nu}=j_{Q}^{\nu}$, reveals that this operator couples the technibaryon to the electromagnetic current. $\partial_{\mu} F^{\mu \nu}$ is therefore proportional to the charge radius operator. This will couple coherently to the nuclear charge. At dimension four the potentially important gauge boson operators are

$$
\begin{array}{ll}
G_{a \mu \nu} G_{a}^{\mu \nu} & G_{a \mu \rho} G_{a \nu}^{\rho} \\
G_{a \mu \nu} \tilde{G}_{a}^{\mu \nu} & G_{a \mu \rho} \tilde{G}_{a \nu}^{\rho}
\end{array}
$$

where $G_{a \mu \nu}$ is the $S U(3)_{C}$ field strength and $\tilde{G}_{a \mu \nu}$ its space time dual. Analogous operators for the electromagnetic field will be less important than the dimension three operator (2.1). The operators (2.2) have the correct Lorentz structure to couple coherently to the entire nucleus at low momentum. However, (2.3) couple to the nuclear spin at low momentum and will lead to scattering rates smaller than from (2.2) by at least $\mathcal{O}\left(A^{2}\right)$, where $A$ is the atomic number.

The lowest dimension operators involving the light quarks are of the form $\bar{q} \Gamma q$, where $\Gamma$ is a Dirac matrix. These arise (aside from (2.1)) from multigluon exchange (analogous to Van der Waals forces) and exchanges of ETC gauge bosons. The former make contributions to the scattering rates suppressed by $\mathcal{O}\left(\alpha_{s}^{2}\right)$. The latter are suppressed by ratios of the technicolor and extended technicolor $\left(\Lambda_{E T C}\right)$ scales. ${ }^{[10]}$ Therefore, only the gauge boson operators (2.1) and (2.2), which give a coherent nuclear coupling, need be considered.

The momentum transfer in nuclear recoil is much less than the nuclear or technibaryon mass. The heavy field formalism is therefore useful in identifying the effective operators for the technibaryons. ${ }^{[11]}$ In the heavy field limit the scalar 
technibaryon fields, $\phi_{v}$, are taken to have definite four velocity $v_{\mu}$. These fields are related to the full field $\phi$ by ${ }^{[10]}$

$$
\phi_{v}(x)=\sqrt{2 M} e^{i M v_{\mu} x^{\mu}} \phi(x)
$$

where $M$ is the technibaryon mass assumed to be of order $\Lambda_{T C}$. The redefinition (2.4) amounts to factoring out of $\phi(x)$ the "fast" space time dependence associated with $M v_{\mu}$ from the "slow" variation associated with the momentum transfer $q_{\mu}=p_{\mu}-M v_{\mu}$. Explicit derivatives, $\partial_{\mu}$, acting on $\phi_{v}(x)$ therefore give factors of $q_{\mu}$. The heavy field formalism gives a convenient parametrization of the nonrelativistic expansion. Lorentz covariant operators are formed from bilinears of the technibaryon fields, $\partial_{\mu}$, and $v_{\mu}$.

The coefficients of the effective operators coupling technibaryons to the gauge fields are not calculable from first principles since the technibaryon is a strongly coupled bound state. The coefficients are therefore estimated using the rules of naive dimensional analysis. ${ }^{[12]}$ Up to dimension seven, the operators coupling the techniboson to the gauge field operators (2.1) - (2.2) are

$$
\begin{gathered}
\frac{e}{\Lambda_{T C}^{2}} \phi_{v}^{*} \phi_{v} v_{\nu} \partial_{\mu} F^{\mu \nu} \\
\frac{g_{s}^{2}}{2 \Lambda_{T C}^{3}} \phi_{v}^{*} \phi_{v} G_{a \mu \nu} G_{a}^{\mu \nu} \\
\frac{g_{s}^{2}}{2 \Lambda_{T C}^{3}} \phi_{v}^{*} \phi_{v} v_{\mu} v_{\nu} G_{a}^{\mu \rho} G_{a}{ }^{\nu} \rho
\end{gathered}
$$

where $\Lambda_{T C}=4 \pi f$, and $f$ is related in a model-dependent way to the electroweak symmetry breaking scale. In most technicolor models, $\Lambda_{T C} \sim 1 \mathrm{TeV}$. As long as the techniboson constituents are charged and colored, (2.5) - (2.7) should give the correct magnitude up to incalculable coefficients of $\mathcal{O}(1)$. For simplicity of notation these $\mathcal{O}(1)$ corrections are absorbed into $\Lambda_{T C}$ below. (2.5) gives a technibaryon charge radius of $r_{c}=\sqrt{6} / \Lambda_{T C}$, while (2.6) and (2.7) contribute to the gluonic polarizabilities. $^{[10]}$ Even though (2.6) and (2.7) are suppressed by an additional power of $\Lambda_{T C}$ relative to (2.5), the relatively large gluonic matrix elements in the nucleus make them potentially important. Consider for example the operator (2.6). The matrix element in the nucleus at zero momentum transfer is related

$\star$ As a "test" of naive dimensional analysis, applying these rules to the neutron gives a charge radius roughly a factor of two smaller than the experimental result. 
via the conformal anomaly to the nucleus mass, $m_{N}$, by $\left\langle N\left|\theta^{\mu}{ }_{\mu}\right| N\right\rangle=-m_{N} \bar{N} N$, where $\theta_{\mu}^{\mu}=-\frac{b g^{2}}{32 \pi^{2}} G_{a \mu \nu} G_{a}^{\mu \nu}$ (ignoring the light quark contribution to $\theta^{\mu}{ }_{\mu}$ ), and $b=11-(2 / 3) n_{\ell}$ is the $\beta$ function coefficient for $n_{\ell}$ light flavors. ${ }^{[13]}$ Using this matrix element, the cross section at small momentum transfer can be compared to the charge radius cross section,

$$
\frac{\sigma_{G G}}{\sigma_{\text {charge radius }}} \simeq \frac{16 \pi^{2}}{b^{2}}\left(\frac{A m_{n}}{Z \alpha \Lambda_{T C}}\right)^{2}
$$

where $m_{n}$ is the nucleon mass. For $\Lambda_{T C} \simeq 1 \mathrm{TeV}$ this ratio is $\sim .1$. The matrix element of (2.7) can be related to the matrix element of the gluon energy momentum tensor. A similar ratio to the charge radius cross section is expected. The charge radius should therefore give the dominant coupling.

In the nonrelativistic limit the differential cross section from (2.5) is

$$
\frac{d \sigma}{d E_{R}}=\frac{16 \pi(Z \alpha)^{2} m_{N}^{2}}{\Lambda_{T C}^{4} E_{R}^{\max }\left(1+m_{N} / M\right)^{2}}\left|F_{c}\left(E_{R}\right)\right|^{2}
$$

where $E_{R}$ is the nuclear recoil kinetic energy in the lab frame, $E_{R}^{\max }=2 m_{N} V^{2} /(1+$ $\left.m_{N} / M\right)^{2}$ is the maximum recoil energy, and $V$ the technibaryon velocity in the lab frame. $F_{c}\left(E_{R}\right)$ is a form factor which accounts for the loss of coherence over the nucleus at finite momentum transfer $\left(\left|F_{c}(0)\right|=1\right)$. For the numerical estimates below a Gaussian distribution of nuclear charge is assumed, $\left|F_{c}\left(E_{R}\right)\right|^{2}=e^{-\frac{2}{3} m_{N} R_{c}^{2} E_{R}}$, with an rms radius $R_{c}=\left(.3+.89 A^{1 / 3}\right) \mathrm{fm} .^{[14]}$ For Ge with a recoil energy of $10 \mathrm{keV}$ (near the threshold energy of the current generation of experiments) $\left|F_{c}\left(E_{R}\right)\right|^{2} \simeq .83$. The differential scattering rate per unit detector mass, $m$, is given by

$$
\frac{d R}{d m d E_{R}}=\frac{\rho}{m_{N} M}\left\langle\frac{d \sigma}{d E_{R}} V\right\rangle
$$

where $\rho$ is the technibaryon density, and

$$
\begin{aligned}
\left\langle\frac{d \sigma}{d E_{R}} V\right\rangle & =\int d V f(V) V \frac{d \sigma}{d E_{R}}\left(V, E_{R}\right) \\
& \equiv V_{0} \frac{d \sigma}{d E_{R}}\left(V_{0}, E_{R}\right)
\end{aligned}
$$

where $f(V)$ is the technibaryon velocity distribution in the lab frame, and $V_{0}$ is a suitably weighted average velocity. The rates from (2.8) are quite small, being 
suppressed by four powers of $\Lambda_{T C}$. Numerically, for Ge

$$
\frac{d R}{d m d E_{R}} \simeq 2 \times 10^{-4}\left|F_{c}\left(E_{R}\right)\right|^{2} \Lambda_{\mathrm{TeV}}^{-4} M_{\mathrm{TeV}}^{-1} \rho_{.3} V_{320}^{-1}(\mathrm{~kg} \mathrm{keV} \mathrm{day})^{-1}
$$

where $\Lambda_{\mathrm{TeV}} \equiv \Lambda_{T C} / \mathrm{TeV}, M_{\mathrm{TeV}} \equiv M / \mathrm{TeV}, \rho_{.3} \equiv \rho /\left(.3 \mathrm{GeV} \mathrm{cm}^{-3}\right)$, and $V_{320} \equiv$ $V_{0} /\left(320 \mathrm{~km} \mathrm{~s}^{-1}\right)$. While this is substantially larger than the rates implied in Ref. [10], it is still well below the current bound for Ge of $\sim 3 /(\mathrm{kg} \mathrm{keV} \mathrm{day})^{-1}{ }^{[7]}$

\section{Fermionic Technibaryons}

In addition to the operator (2.1), technibaryons with spin can couple to the dimension two electromagnetic field strength, $F^{\mu \nu}$, via dipole moments. In virtually any conceivable technicolor scenario, some of the technibaryon constituents will be charged, and such couplings will arise. This leads to much more dramatic effects.

For definiteness spin $\frac{1}{2}$ technibaryons will be considered. The extension to higher spin is straightforward. Again, the heavy field formalism is useful for writing the effective operators. The technifermion field of definite four velocity, $\Psi_{v}$, is related to the full field $\Psi$ by ${ }^{[11]}$

$$
\Psi_{v}(x)=e^{i M \psi \cdot x} \Psi(x)
$$

At dimension five the only operator suppressed by a single power of the technicolor scale is the magnetic moment

$$
\frac{e}{\Lambda_{T C}} \epsilon_{\mu \nu \rho \sigma} v^{\rho} \bar{\Psi}_{v} S^{\sigma} \Psi F^{\mu \nu}
$$

where $S^{\mu}$ is the spin operator. ${ }^{[1,15]}$ The coefficient is taken from the rules of naive dimensional analysis. This gives the technifermion a magnetic moment $\mu=e / \Lambda_{T C}$.

The technifermion magnetic moment couples to the nuclear magnetic moment and coherently to the current produced by the nuclear charge (in the technifermion rest frame). ${ }^{[16]}$ The differential cross section in the nonrelativistic limit is

$$
\begin{aligned}
\frac{d \sigma}{d E_{R}} & =\frac{4 \pi \alpha^{2}}{\Lambda_{T C}^{2}\left(1+m_{N} / M\right)^{2} E_{R}^{\max }}\left\{\frac{2(J+1)}{3 J}\left(\frac{\mu A}{\mu_{n}}\right)^{2}\left|F_{s}\left(E_{R}\right)\right|^{2}\right. \\
& \left.+Z^{2}\left(\left(1+m_{N} / M\right)^{2} \frac{E_{R}^{\max }}{E_{R}}-\frac{2 m_{N}}{M}-1\right)\left|F_{c}\left(E_{R}\right)\right|^{2}\right\}
\end{aligned}
$$

where $J$ is the nuclear spin, $\mu / \mu_{n}$ is the nuclear magnetic moment in units of the nuclear Bohr magneton $\left(=e / 2 m_{n}\right)$, and $F_{s}\left(E_{R}\right)$ is a form factor for the nuclear 
spin. For the numerical estimates below the somewhat crude assumption is made of a Gaussian distribution of spin within the nucleus with spin radius $R_{s}=1.25 R_{c}{ }^{[14]}$ For large recoil energy the nuclear magnetic moment and coherent contributions are typically the same order. The coherent contribution has an infrared singularity, giving a small enhancement for experimentally accessible recoil energies. For Ge in natural abundance, the coherent nuclear coupling dominates. Numerically, for $E_{R}<<E_{R}^{\max }$,

$$
\frac{d R}{d m d E_{R}} \simeq 1 \times 10^{-2} \frac{E_{R}^{\max }}{E_{R}}\left|F_{c}\left(E_{R}\right)\right|^{2} \Lambda_{\mathrm{TeV}}^{-2} M_{\mathrm{TeV}}^{-1} \rho_{.3} V_{320}^{-1}(\mathrm{~kg} \mathrm{keV} \text { day })^{-1}
$$

For $E_{R}=10 \mathrm{keV}, d R /\left(d m d E_{R}\right) \simeq .15 \Lambda_{\mathrm{TeV}}^{-2} M_{\mathrm{TeV}}^{-1} \rho_{.3} V_{320}^{-1}(\mathrm{~kg} \mathrm{keV} \text { day })^{-1}$. Scattering rates of this order may be accessible to the next generation of Ge experiments. ${ }^{[17]}$

The coupling to the nuclear magnetic moment can be significant in elements with large magnetic moments. For ${ }^{93} \mathrm{Nb}$ with $J=\frac{9}{2}$ and $\mu \simeq 6.17 \mu_{n}$, the magnetic moment gives the dominant contribution even for $E_{R}=10 \mathrm{keV}$. Numerically,

$$
\frac{d R}{d m d E_{R}} \simeq 2\left|F_{s}\left(E_{R}\right)\right|^{2} \Lambda_{\mathrm{TeV}}^{-2} M_{\mathrm{TeV}}^{-1} \rho_{.3} V_{320}^{-1}(\mathrm{~kg} \mathrm{keV} \text { day })^{-1}
$$

This is comparable to the scattering rate for a technibaryon with weak isospin. The large magnetic moment makes $\mathrm{Nb}$ a good candidate material to search for dark matter technifermions. The nuclear magnetic moment scattering is also significant for $\mathrm{NaI}$ and $\mathrm{CaF}_{2}$.

In addition to the magnetic dipole moment (3.2), a technifermion can possess an electric dipole moment. This $\mathrm{P}$ and $\mathrm{T}$ violating operator should be suppressed by the scale associated with $\mathrm{CP}$ violation, $\Lambda_{C P}$. In a realistic model this is presumably related to an extended technicolor scale responsible for light fermion masses. If the lowest dimension $\mathrm{P}$ and $\mathrm{T}$ violating operators among the technibaryon constituents are four-Fermi type of dimension six, the electric dipole moment must be suppressed by two powers of $\Lambda_{C P}$. Including this suppression then gives the estimate

$$
\frac{e}{\Lambda_{T C}}\left(\frac{\Lambda_{T C}}{\Lambda_{C P}}\right)^{2} v_{\mu} 2 \bar{\Psi}_{v} S_{\nu} \Psi_{v} F^{\mu \nu}
$$

where the coefficient of the $\mathrm{CP}$ violating dimension six operator is absorbed in $\Lambda_{C P}$. This gives the technifermion an electric dipole moment $d=e \Lambda_{T C} / \Lambda_{C P}^{2}$. In 
the nonrelativistic limit, the differential cross section is

$$
\frac{d \sigma}{d E_{R}}=\frac{8 \pi(Z \alpha)^{2}}{\left(\Lambda_{C P}^{2} / \Lambda_{T C}\right)^{2}\left(1+m_{N} / M\right)^{2} E_{R}^{\max }} \frac{m_{N}}{E_{R}}\left|F_{c}\left(E_{R}\right)\right|^{2}
$$

The ratio of electric dipole scattering to the coherent part of the magnetic dipole scattering is $\mathcal{O}\left(m_{N} \Lambda_{T C}^{4} / E_{R}^{\max } \Lambda_{C P}^{4}\right)$. The enhancement occurs because the electric dipole couples directly to the nuclear charge rather than the nuclear current. Roughly, for $\Lambda_{C P}<30 \Lambda_{T C}$ the electric dipole is more important. Numerically, for Ge

$\frac{d R}{d m d E_{R}} \simeq 1 \times 10^{4} \frac{E_{R}^{\max }}{E_{R}}\left(\frac{\Lambda_{T C}}{\Lambda_{C P}}\right)^{4}\left|F_{c}\left(E_{R}\right)\right|^{2} \Lambda_{\mathrm{TeV}}^{-2} M_{\mathrm{TeV}}^{-1} \rho_{.3} V_{320}^{-1}(\mathrm{~kg} \mathrm{keV} \text { day })^{-1}$

With $E_{R}=10 \mathrm{keV}$, the current bound from Ge already gives a limit of $\Lambda_{C P}>$ $14\left(\Lambda_{\mathrm{Tev}}^{2} \rho_{.3} / M_{\mathrm{TeV}} V_{320}\right)^{1 / 4} \mathrm{TeV}$.

\section{Conclusions}

The detectability of singlet dark matter technibaryons depends sensitively on the spin. For scalar technibaryons the dominant coupling is through the charge radius. The rate from this coupling is suppressed by four powers of the technicolor scale. The total scattering rate for Ge, assuming a threshold recoil energy of $5 \mathrm{keV}$, is $\sim 1 \times 10^{-2}(\mathrm{~kg} \text { day })^{-1}$ for $\Lambda_{T C} \simeq 1 \mathrm{TeV}$. While this is well beyond the reach of current experiments, it is comparable to spin dependent scattering rates for the neutralino of supersymmetry. ${ }^{[14,18]}$ For fermionic technibaryons the dominant coupling is through the magnetic moment. The scattering rate is suppressed by two powers of the technicolor scale. The total rate can be sizeable. For Ge in natural abundance, assuming a threshold of $5 \mathrm{keV}$, the rate is $\sim 3(\mathrm{~kg} \text { day })^{-1}$ for $\Lambda_{T C} \simeq 1$ $\mathrm{TeV}$. This is comparable to the spin independent rates for the neutralino. ${ }^{[14,18]}$ Even larger rates are attainable in elements with sizeable nuclear magnetic moments. For $\mathrm{Nb}$, again assuming a threshold energy of $5 \mathrm{keV}$, the total rate is $\sim 35(\mathrm{~kg}$ day $)^{-1}$. In principle, a technibaryon electric dipole moment could also lead to sizeable rates. However, in a "realistic" model with $\Lambda_{C P} \sim \Lambda_{E T C} \sim 10^{3} \Lambda_{T C}$, the magnetic moment still dominates the scattering.

An important feature of singlet technibaryon scattering is that the dominant couplings are electromagnetic. This is in contrast to the spin independent coupling of the neutralino which is typically dominated by operators such as (2.2). In principle then, comparison of scattering rates in different materials could allow the technibaryon to be distinguished from the neutralino. In addition, the coherent 
part of the magnetic moment scattering has a distinctive energy dependence at low energy (Eq. (3.3)). Finally, it is worth noting that the analysis presented here is generally applicable to any dark matter particle carrying a quantum number for a continuous symmetry, but which is a singlet under the standard model gauge groups. $^{[4]}$

\section{REFERENCES}

1. See, for example, the collection of articles in Particle Physics and Cosmology: Dark Matter, edited by M. Srednicki, (North Holland, Amsterdam, 1990).

2. S. Nussinov, Phys. Lett. B 165 (1985) 55; R. Chivukula and T. Walker, Nucl. Phys. B 329 (1990) 445.

3. S. Barr, R. Chivukula, and E. Farhi, Phys. Lett. B 241 (1990) 387.

4. D. Kaplan, Phys. Rev. Lett. 68 (1992) 741. This paper discusses more general models with dark matter candidates carrying anomalous U(1)'s.

5. P. Smith et. al.,, Nucl. Phys. B 206 (1982) 333; T. Memmick et. al., Phys. Rev. D 41 (1990) 2074; P. Verkerk et. al., Phys. Rev. Lett. 68 (1992) 1116.

6. G. Starkman, A. Gould, R. Esmailzadeh, and S. Dimopolous, Phys. Rev. D 41 (1990) 3594; D. Caldwell, in Proceedings of the International School of Astroparticle Physics, edited by D. Nanopoulos (World Scientific, Singapore, 1991) p. 375.

7. S. Ahlen, et. al., Phys. Lett. B 195 (1987) 60; D. Caldwell, et. al., Phys. Rev. Lett. 61 (1988) 510; D. Reusser, et. al., Phys. Lett. B 255 (1991) 143.

8. S. Nussinov, Phys. Lett. B 179 (1986) 103.

9. S. Nussinov, Phys. Lett. B, 279 (1992) 111.

10. R. Chivukula, A. Cohen, M. Luke, and M. Savage, Phys. Lett. B 298 (1993) 380 .

11. H. Georgi, Phys. Lett. B 240 (1990) 447.

12. A. Manohar and H. Georgi, Nucl. Phys. B 234 (1984) 189.

13. M. Shifman, A. Vainshtein, V. Zakharov, Phys. Lett B 78 (1978) 443.

14. J. Ellis and R. Flores, Phys. Lett. B 263 (1991) 259; J. Ellis and R. Flores, Phys. Lett. B 175 (1993) 175.

15. E. Jenkins and A. Manohar, Phys. Lett. B 255 (1991) 558.

16. Aspects of the low energy magnetic dipole moment scattering have been considered in a different context by S. Raby and G. West, Phys. Lett. B 200 (1988) 547. 
17. B. Young, et. al., in Proceedings of the XXVI International Conference on High Energy Physics, edited by J. Sanford (American Institute of Physics, New York, 1993) p. 1260.

18. M. Goodman and E. Witten, Phys. Rev. D 31 (1985) 3059; K. Griest, Phys. Rev. D 38 (1988) 2357. For recent calculations including constraints from

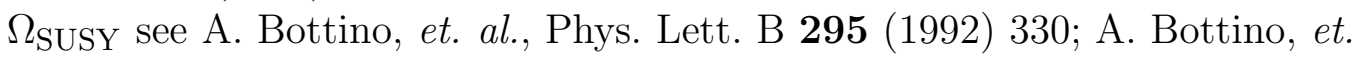
al., preprint DFTT 38/93; M. Drees and M Nojiri, preprint MAD/PH/768. 